\title{
Análisis de las concepciones de la masculinidad a la base de la intervención en hombres propuesta por el plan de seguridad pública del Ministerio del Interior
}

\author{
Analysis of the conceptions of masculinity that give base \\ to men interventions, as proposed by the public security \\ plan of the Department of the Interior
}

\author{
Gabriel Abarca Brown ${ }^{I}$ \\ Carla Carvajal Fuentes ${ }^{2}$ \\ Ángela Cifuentes Astete ${ }^{3}$
}

Resumen

El presente artículo tiene por objetivo analizar las concepciones de masculinidad a la base de una intervención propuesta por el Ministerio del Interior para el trabajo con hombres que ejercen violencia en contextos de pareja, a la luz de los Estudios de la(s) Masculinidad(es) y los aportes de la Teoría Queer. Mediante el análisis de contenido se constatan concepciones esencialistas y normativas, amparadas en la heteronormatividad de los sexos. Asimismo, se evidencia la superposición de discursos que la sitúan como dispositivo jurídico de control, por una parte, y como espacio clínico psicoterapéutico, por otra. Finalmente, se destaca la contribución de los estudios de la(s) masculinidad(es) que incorporan definiciones semióticas al análisis.

Palabras clave: Masculinidad(es), Heteronormatividad, Violencia en la pareja, Plan de seguridad pública.

1 Docente Escuela de Psicología de la Universidad de Santiago de Chile. E-mail: gabriel.abarca@ usach.cl

2 Psicóloga del Programa Vínculo, Ilustre Municipalidad de Recoleta. E-mail: carla.carvajalf@gmail.com

3 Psicóloga de la Comunidad Terapéutica: Centro de Tratamiento Integral Orión Mujeres. E-mail: angela.cifuentes@gmail.com 


\begin{abstract}
This article analyzes the conceptions of masculinity that ground an intervention proposed by the Department of the Interior, which is addressed to men that commit violent acts against women who are their partners. This study is made through Masculinity(ies) Studies and contibutions of Queer Theory. Through Content Analysis, conceptions of normative and essentialist masculinity and hegemonic heteronormativity of the sexes are revealed. The conceptions of the intervention show a superimposition of discourses that place it as a legal control tool, as well as a psychotherapeutic clinical space. Finally, other contributions of masculinity(ies) studies, that include semiotic definitions to the analysis, are highlighted.
\end{abstract}

Key words: Masculinity(ies), Heteronormativity, Violence within partners, Public Security Plan.

\title{
Problematización
}

Los universos subjetivos de lo masculino y lo femenino han sufrido impactos significativos durante los últimos siglos. La permeabilidad de los límites entre lo público y lo privado, los métodos de contracepción y los actuales avances en fertilización in vitro y manipulación genética han permitido el acceso de la mujer a instancias de poder e interpelado el lugar atribuido a los hombres históricamente (Castells, 2001).

El devenir de dichos cambios ha cuestionado la posición del hombre-patriarca al interior de la familia, y por tanto, a la familia nuclear tradicional en sí. Tal como señala Olavarría (2000), ni el hombre cumpliría una función principal, ni la mujer e hijos(as) un papel secundario.

Dentro de este escenario, las articulaciones y expresiones de la subjetividad masculina se han constituido como objeto de estudio para las ciencias sociales -y en especial para los estudios de género- al destacar la "crisis" de ejes constitutivos de la identidad de los hombres, a saber: los roles de proveedor, protector y reproductor (Burín, 2007). 
Para los estudios de la(s) masculinidad(es) - de reciente data dentro de los estudios de género ${ }^{4}$ - la "crisis" de la masculinidad correspondería a la transformación de un modelo hegemónico de masculinidad, que cuestiona un esquema culturalmente construido en el que los hombres son asociados con atributos como independencia, agresividad y conductas violentas (De Keijzer, 2001).

A pesar de las diversas expresiones de la masculinidad -paternidad, salud sexual, salud reproductiva, etc.- la violencia contra la mujer ha sido uno de los principales objetos de análisis para los estudios de género. Su comprensión radicaría en que los valores patriarcales -ahora en crisis- han sentado las bases para que los hombres dominen y ejerzan control sobre las mujeres (Zarza \& Frojan, 2005).

El ejercicio de la violencia sería, por décadas, una cualidad asignada socioculturalmente a los hombres, utilizándola en función de legitimar el poder que poseen en el marco de las relaciones de género (Bourdieu, 2000), a partir de la posición hegemónica que detentan respecto a las mujeres y las masculinidades consideradas subordinadas. De esta forma, el fenómeno de la violencia contra la mujer en la actualidad estaría ligado a la transformación que padece la institución familiar y la masculinidad hegemónica, generada por las contradicciones sociales, de clase y por los cambios acontecidos durante el siglo XX en las sociedades modernas (Ferrer \& Bosch, 2005).

En este sentido, Amuchástegui (2008) sostiene que ya sea por los estudios de género, los movimientos sociales o el desarrollo científico en las últimas décadas, se ha instalado en el debate a un "sujeto apto para la intervención social: el hombre violento, delineado a contraluz de su complemento, la mujer maltratada" (p. 55). A la vez, se ha hecho visible la necesidad de contar con instrumentos analíticos que permitan explicar los comportamientos observados, así como prevenirlos y estimular su modificación hacia nuevas formas de relación entre hombres y mujeres.

Fue a contar de 1995, durante las conferencias sobre Población y Desarrollo del Cairo, y de Beijing sobre la Mujer, donde se enfatizó la

4 Los estudios de la(s) masculinidad(es) surgen en torno a la década de los 80 . 
relevancia de analizar la responsabilidad de los hombres en la violencia contra las mujeres, reafirmando que "para lograr los objetivos de un desarrollo sustentable, un componente esencial es el logro de la igualdad entre los géneros con la participación de los varones en estos ámbitos” (Valdés, 2000, p. 6, en Olavarría, 2008).

Para el caso chileno, las intenciones planteadas a nivel internacional en torno al trabajo con hombres no habrían llegado a cumplirse. En términos generales, Aguayo y Sadler (2010) refieren que los hombres, tanto en las políticas como en la mayoría de las discusiones, son etiquetados como violentos, alcohólicos, drogadictos y delincuentes, en oposición a personas con necesidades en salud mental y vulnerabilidades psicosociales. Al mismo tiempo, han sido dejados de lado en el debate en torno al género al priorizarse la intervención con mujeres y niños(as).

En cuanto a los programas en violencia hacia la mujer, los mismos autores refieren que si bien existen intervenciones diseñadas para aumentar la conciencia de los actos de violencia y proporcionar apoyo a las víctimas, estas han sido inadecuadas a partir de sus conceptualizaciones. Por otro lado, plantean que algunos fondos públicos han entregado los primeros servicios para hombres que ejercen violencia -principalmente de psicoterapia de grupos- los que, sin embargo, no han sido sistematizados o sus resultados no se encuentran disponibles.

Una de las recientes iniciativas en materia de violencia en Chile es la "Intervención en hombres que cometen actos de violencia contra la mujer en el contexto de las relaciones de pareja”, impulsada por el Plan de Seguridad Pública del Ministerio del Interior de Chile. La propuesta sostiene como fundamento que la violencia manifestada en espacios privados incide en la producción de conductas violentas y delictuales en los espacios públicos; lo que enmarca a la intervención con hombres en el contexto de la seguridad pública, y no dentro de políticas que consideren la salud o la vulnerabilidad psicosocial de manera explícita y directa.

Es frente al etiquetamiento que han sufrido las masculinidades y la escasa oferta de programas para hombres que ejercen violencia contra la mujer, que el objetivo central de este artículo será analizar las concepciones de masculinidad a la base de las Orientaciones Técnicas para el año 2010, 
de la "Intervención en Hombres que Cometen Actos de Violencia Contra la Mujer en el Contexto de Pareja”, presentada por el Plan de Seguridad Pública del Ministerio del Interior, a la luz de los Estudios de la(s) Masculinidad(es) y los aportes de la Teoría Queer. Asimismo, resulta de interés para este trabajo, por una parte, indagar en las posibles ventajas y desventajas que ofrecen la concepción de masculinidad para el trabajo con hombres que ejercen violencia y, por otra, discutir sobre las ventajas y desventajas de comprender la masculinidad y la violencia hacia la mujer dentro del marco de la política de seguridad pública.

Resulta relevante llevar a cabo este análisis principalmente por dos motivos: en primer lugar, es necesario poner en debate las bases sobre qué se entiende por masculinidad(es) en las primeras intervenciones realizadas por el Estado en materia de violencia contra la mujer, pues esta comprensión permitiría conocer los alcances y obstáculos en el trabajo con sujetos victimarios. En segundo término, no abordar este tipo de intervenciones contribuiría al silenciamiento que han sufrido los hombres y las masculinidades en cuanto a políticas de género se refiere, las cuales han sido dirigidas principalmente a niños(as) y mujeres, y a los efectos que la violencia ejercida por hombres ha generado.

Masculinidad en crisis: el hombre como objeto de estudio

Diversos cambios socioculturales acontecidos en las últimas décadas han redefinido las relaciones de género $y$, consecuentemente, las categorías de femineidad y masculinidad. Sin embargo, el abordaje de la masculinidad desde las ciencias sociales emerge recientemente en la historia.

A partir de la década de los setenta y ochenta surge una proliferación de estudios que buscan denunciar y destituir los modelos tradicionales instituidos (Burín, 2007), haciendo visible la crisis del sistema sexo/ género (Olavarría, 2003). En este sentido, los estudios develan la crisis de la lógica de la diferencia sexual, específicamente respecto a sus principios esencialistas, naturalistas, biologicistas, de individualismo y a-historicidad (Burín, 2007). 
No obstante, los hombres se constituyen en objeto de estudio marcadamente en la década de los noventa (Burín, 2007), debido a la intensa pérdida del poder económico, la transformación del trabajo, los cambios profundos en la clásica familia nuclear patriarcal, además de la lucha de las mujeres y su incorporación a nuevas modalidades laborales (Burín, 2007; Castells, 2001; Olavarría, 2003), fenómenos que han incidido en las subjetividades, resquebrajando sus antiguas bases identitarias (Burín, 2007). Asimismo, surgen los primeros cuestionamientos sobre la identidad y búsqueda de rompimiento con la masculinidad hegemónica (Abarca, 2000; De Keijzer, 2001; Montecino, 1996), emergiendo demandas desde movimientos homosexuales por el reconocimiento de sus derechos y de la diversidad (Olavarría, 2003).

Consecuentemente, los estudios de la masculinidad muestran que es necesario considerar la existencia de diferentes masculinidades (Araujo \& Rogers, 2000), donde la comprensión de sus transformaciones no se sitúa como un objeto de estudio aislado, sino como parte de una estructura mayor (Connell, 1997), constituyéndose el mundo contemporáneo en un escenario altamente cambiante e inestable que provoca acelerados cambios en las relaciones genéricas (De Keijzer, 2001; Burín, 2007) y por tanto, en la(s) masculinidad(es) en tanto posición subjetiva.

De esta manera, los estudios han trazado una crisis supeditada a la ruptura de ciertos sustentos estructurales, acompañadas por una pérdida de paradigmas acerca del significado de la masculinidad (Araujo \& Rogers, 2000); crisis que se ve confrontada entre los viejos referentes simbólicos y los actuales (Montesinos, 2002) donde la masculinidad hegemónica facilita el ejercicio y la expresión del poder de los sujetos hombres al mismo tiempo que los constriñe -particularmente en el espacio privado- debido a lo inalcanzable del modelo-imagen masculino (Abarca, 2000; Garda, 1998).

Es relevante precisar desde una noción relacional de la categoría género (Scott, 1997) que no solo entra en crisis la masculinidad, sino el conjunto de formas de vida -de hombres y mujeres- que estructuró el siglo XX (Olavarría, 2003). En otras palabras, es una crisis de las relaciones de género, que para los varones se manifiesta como crisis de la masculinidad (Connell, 1997; Olavarría, 2003). 
Concepto de Masculinidad desde los estudios de la(s) masculinidad(es)

El diccionario de la Real Academia Española define el concepto masculinidad en tres términos: referido a aquel ser dotado de órganos para fecundar; como perteneciente o relativo a este ser; y en tanto cualidades como varonil y enérgico (Real Academia Española, 2001). Tales acepciones dan cuenta del uso moderno del concepto masculinidad en la cultura occidental, denotando su característica relacional inherente-masculinidad en contraste con la femineidad-y adjudicando a la conducta -violenta, dominante, activa- el rasgo que define el tipo de sujeto considerado masculino (Connell, 1997).

Según Connell (1997) es posible distinguir cuatro enfoques que han empleado estrategias diferentes para tipificar lo masculino en una persona. Tales enfoques, si bien tienden a combinarse en la práctica, son reconocibles nítidamente respecto a su lógica:

- Enfoque esencialista: un rasgo o una serie de rasgos de la vida de los hombres define el núcleo de lo masculino. Por ejemplo, considerar la agresividad como intrínsecamente masculina. No obstante, la delimitación de la esencia es bastante arbitraria, lo que genera disidencias entre los mismos teóricos esencialistas.

- Definición de la ciencia social positivista: la masculinidad se conceptualiza como "lo que los hombres realmente son". Un ejemplo son las escalas masculinidad/femineidad, validadas estadísticamente en psicología. Surgen tres dificultades. En primer lugar, como señala la epistemología moderna, no hay descripción sin punto de vista, por tanto lo que aparece como neutral, supone asunciones subyacentes sobre el género. Segundo, el enfoque positivista descansa en tipificaciones de género propias del sentido común. Tercero, considera solo las diferencias entre hombres y mujeres como grupo, y no considerando cómo los hombres difieren entre ellos y las mujeres entre ellas (por ejemplo, mujeres masculinas, hombres femeninos). 
- Definiciones normativas: la masculinidad es definida como norma social para el comportamiento de los hombres. Se le critica a las teorías de roles sexuales que a menudo mezclan definiciones normativas con definiciones esencialistas. Además, estas definiciones no tienen asidero sobre la masculinidad al nivel de la personalidad, por lo que se hace insostenible la correspondencia entre rol e identidad.

- Enfoques semióticos: basándose en la lingüística estructural, este enfoque define la masculinidad a partir de un sistema de diferencia simbólica, donde masculinidad se conceptualiza como no-femineidad. En la oposición semiótica, la masculinidad es el lugar de la autoridad simbólica y la femineidad es simbólicamente definida por la carencia, siendo el falo la propiedad significativa. Este enfoque se ha aplicado en los análisis culturales feministas, análisis postestructuralistas de género, y en el psicoanálisis, en los estudios de simbolismo lacanianos. De acuerdo a Connell (1997), este enfoque ha sido muy efectivo en el análisis cultural al cuestionar el esencialismo y lo paradojal de las definiciones positivistas y normativas.

Connell (1997) agrega que para contemplar la amplitud de tópicos respecto a la conceptualización de la masculinidad se debe recurrir a otros elementos que atraviesan las relaciones (aspectos sociales, económicos, geográficos, entre otros). De esta forma, describe a la masculinidad como "la posición en las relaciones de género, las prácticas por las cuales los hombres y mujeres se comprometen con esa posición de género, y los efectos de estas prácticas en la experiencia corporal, en la personalidad y en la cultura" (p. 35).

Desde un discurso postestructuralista, una de las impulsoras de la Teoría Queer, Judith Butler (2001) critica los conceptos esencialistas, y por tanto, a los mandatos de heterosexualidad normativa. La categoría 
"mujeres" -que indica una identidad común- la conceptualiza como normativa y excluyente, debido a que su empleo no considera que el género se constituye de diversas formas, dependiendo de contextos históricos distintos, y que es atravesado por particularidades raciales, de clase, étnicas, sexuales y regionales de identidades discursivamente constituidas.

Asimismo, Butler (1990) pone en cuestión la diferenciación entre sexo y género existente en las diversas perspectivas. Parafraseando a Foucault, señala que la noción de una verdad acerca del sexo es creada por prácticas reguladoras que producen identidades acordes a reglas impuestas por la matriz cultural, en la cual ciertos tipos de identidades no tienen cabida: "aquellas en las que el género no es consecuencia del sexo y otras en las que las prácticas del deseo no son consecuencia ni del sexo ni del género" (Butler, 2001, p. 72). Es decir, la heterosexualización del deseo instituye la producción de asimetrías y oposiciones entre lo femenino y masculino en tanto condiciones que distinguen a hombres y mujeres.

Por tanto, el sexo -considerado en los discursos hegemónicos como una "sustancia" que define la masculinidad- es "una significación performativamente realizada (y, por tanto, que no 'es')" (Butler, 2001, p. 99). Es decir, para Butler el género es esencialmente una identificación -una fantasía dentro de otra fantasía-, que se articula en lo que denomina el performance, esto es, la repetición que imita constantemente las significaciones (Butler, 2006; Fonseca \& Quintero, 2009).

Bajo este punto de vista, el sexo sería un efecto performativo de los discursos de la modernidad, donde se han inscrito ilusiones acerca de la identidad y nociones de género reificadas que sustentan el poder heterosexista. En este sentido, las definiciones esencialistas y positivistas planteadas por Connell (1997) darían cuenta de la regulación heteronormativa (Butler, 2001).

5 Butler (2006) concibe la noción de género en tanto acto performativo, es decir, como una actuación reiterada y obligatoria en función de normas sociales que exceden y anteceden a los sujetos. De esta forma, Butler situará la reproducción de normas hegemónicas al interior de la relación entre los géneros. 
Conceptualización de la violencia en contextos de pareja desde el enfoque de género y los estudios de la/s masculinidad/es

En las sociedades patriarcales las posiciones subjetivas hegemónicas relativas al género - masculino y femenino- se articularían posicionando al hombre por sobre la mujer, al destacar el poder y la autoafirmación como rasgo inherente de los varones (Bonino, 2009).

Para Badinter (1992, en Sadler, 2007), este modelo de masculinidad ofrecería ciertos mandatos sobre cómo ser hombre, los cuales incidirían en la subjetividad a tono de imperativo: no eres mujer; no eres bebé; y por último, no eres homosexual. Sin embargo, si bien estos mandatos sitúan al hombre en una posición de poder, también suponen comportamientos autodestructivos y generadores de violencia hacia ellos mismos, hacia masculinidades subordinadas, hacia las mujeres y los/as niños/as (Olavarría, 2000; Sadler, 2007). En otros términos, los comportamientos amparados en el modelo hegemónico de masculinidad establecen un costo tanto para la salud de los hombres como para quienes los rodean.

A partir de la prevalencia de un modelo hegemónico, Connell (1997) refiere que pueden derivarse dos patrones de violencia: uno en que muchos miembros del grupo privilegiado usan la violencia para sostener su dominación, amparados en la ideología de supremacía, con expresiones que van desde un "silbido de admiración (...) hasta el asesinato por el dueño patriarcal de la mujer" (p. 44); y otro, en que la violencia llega a ser importante en la política de género entre los hombres. Gran parte de los episodios de violencia mayor dan cuenta de aquello (combates militares y asaltos armados), donde dichos eventos son transacciones entre hombres.

El comportamiento violento de los hombres, desde los estudios de la(s) masculinidad(es), es visto como una maniobra con la que no solo los varones buscan instalar una situación favorable de poder, sino también reafirmar su identidad masculina, asentada en la creencia patriarcal de superioridad. Mantener bajo dominio a la mujer le permite, además, controlar las emociones que esta le provoca: temor, dependencia, envidia, etc. (Bonino, 1990, en Bonino, 2009). 
Fernández-Llebrez (2005) en una revisión de diversos autores, destaca como rasgos psicosociales presentes en hombres que ejercen violencia: una clara búsqueda del control de la relación; dificultad para hacer frente de forma adecuada a situaciones conflictivas, sobre todo de índole personal; conductas violentas mayoritariamente solo en el ámbito familiar; una tendencia a ver como legítima la violencia a la hora de resolver los conflictos; formación en una cultura de la desigualdad sobre lo que debe ser un hombre y una mujer; poca o nula capacidad para comunicar sus sentimientos y para hablar de sus problemas afectivos aislamiento emocional y evitación de la intimidad-; inseguridad tapada de autoritarismo y su correspondiente fuerte dependencia emocional; poca tolerancia a la frustración, temor a perder autoestima y poder; pobre imagen de sí mismo; y por último, han vivido en un ambiente familiar sobreprotector y permisivo.

Cabe señalar que Fernández-Llebrez (2005) observa el alto porcentaje de abuso de alcohol y otras sustancias presente en hombres violentos como un factor de riesgo y no explicativo de esta conducta; así como también descarta los casos en que la violencia responde a efectos de trastornos mentales, que representan un 10\%.

Por tanto, tal como señala De Keijzer (1997), si bien no todos los hombres son violentos, una gran parte de estos no toma conciencia de las agresiones dirigidas hacia las mujeres, debido a que la violencia estaría normalizada y legitimada por los valores patriarcales que configuran la masculinidad hegemónica. La complicidad masculina daría lugar al silencio, incluso al interior de las propias familias.

Conceptualización sobre la masculinidad y la violencia en contextos de pareja desde la intervención con hombres planteada por el Plan de Seguridad Pública ${ }^{6}$

El Ministerio del Interior, a través del Plan Comunal de Seguridad Pública y sus Fondos de Apoyo a la Gestión Municipal (FAGM), ha

6 Intervención contenida en las Orientaciones Técnicas del Fondo de Apoyo a la Gestión Municipal (FAGM), en el eje de Rehabilitación y Reinserción Social (Ministerio del Interior, 2010). 
impulsado la "Intervención en hombres que cometen actos de violencia contra la mujer en el contexto de relaciones de pareja”, como una de las recientes propuestas en cuanto a violencia se refiere.

El objetivo de la intervención -expuesto en las Orientaciones Técnicas (OT) - es "disminuir las conductas de violencia contra la mujer cometidas por hombres en el contexto de las relaciones de pareja (actual o pasada) a través del abordaje terapéutico especializado en población masculina de bajo riesgo o riesgo moderado" (Ministerio del Interior, 2010, p. 224). Asimismo, agrega: "el objetivo del tratamiento buscará principalmente un cambio concreto en la conducta agresora que presenten los sujetos, sin perjuicio que, como consecuencia de esta misma intervención, se generen otros cambios favorables en la persona atendida” (Ministerio del Interior, 2010, p. 221).

La intervención se fundamenta, por una parte, en que "la violencia contra la mujer constituye una de las formas más preocupantes de vulneración de derechos humanos de estas", y por otra, en que "la Política de Seguridad Ciudadana de Chile reconoce que la violencia que se expresa en los espacios privados aporta a la producción de conductas violentas y/o delictuales en los espacios públicos" (Ministerio del Interior, 2010, p. 217).

La población objetivo a la que está orientada la intervención está delimitada por criterios de inclusión mínimos, entre los que se consideran: ser hombre, mayor de 14 años y ejercer violencia circunstancial o considerada de riesgo leve-moderado en el contexto de pareja. Dichos beneficiarios pueden ser derivados desde diferentes fuentes, a saber: sistema judicial, red de salud y territorial, o por consulta espontánea de los usuarios (Ministerio del Interior, 2010).

Dentro de los principios orientadores, la intervención destaca la complejidad de las dinámicas de violencia que experimentan las familias en el contexto de las relaciones de pareja, así como las diferencias en su manifestación. La violencia contra la mujer es vista como un fenómeno que responde a aspectos tanto culturales como propios del proceso de socialización -caracterizado principalmente, por una distribución no equitativa del poder entre géneros-, que ha derivado en el aprendizaje y utilización de patrones cognitivos y relacionales que favorecen la violencia. 
Según la evidencia empírica que avala este tipo de proyectos, las intervenciones son eficaces en la medida que se integran el "análisis funcional del comportamiento violento, los aspectos cognitivos y relacionales que sustentan la violencia contra la mujer, el enfoque de género y la temática de la masculinidad entendida desde la necesidad de redefinir el rol masculino tras la 'pérdida de poder' que ha significado para muchos hombres el progresivo empoderamiento de la mujer" (Ministerio del Interior, 2010, p. 217).

En este sentido, para las OT, la redefinición del rol masculino en el marco de las relaciones de pareja es crucial. La intervención sostiene que en:

ciertos estudios sobre la violencia contra la mujer, basados en el enfoque de género, han asociado algunas manifestaciones de violencia con eventuales dificultades de los hombres para redefinir el rol masculino en un contexto social y cultural cambiante, en particular ante la constatación del progresivo empoderamiento que ha experimentado la mujer (...) este proceso ha provocado que, de hecho, muchas mujeres logren ejercer de manera efectiva sus derechos, más allá de las expectativas de hombres socializados en el marco de una matriz cultural patriarcal, lo cual tensiona la forma en que estos hombres comprenden su propio rol en la relación de pareja (Ministerio del Interior, 2010, p. 223).

De acuerdo a lo anterior, la violencia contra la mujer es asumida no como producto de un "trastorno mental" o "enfermedad psiquiátrica", por lo que el tratamiento a los hombres persigue alcances terapéuticos limitados al análisis y modificación funcional, orientado de manera pragmática a la corrección del comportamiento violento y al desarrollo de competencias sociales y relacionales necesarias para el desarrollo de vínculos no violentos. Por esta razón no se concibe como una intervención estrictamente clínica.

Por último, el enfoque de tratamiento que asume la intervención contempla tres lineamientos. En primer lugar, el Modelo Feminista-Psicoeducacional, extraído del Duluth Domestic Abuse Intervention Project Program de Minnesota, postula que una de las principales causas de la vio- 
lencia doméstica radica en la ideología patriarcal y la complicidad de la sociedad que brinda a los hombres la oportunidad del ejercicio de poder por sobre las mujeres. En segundo término, el Modelo Cognitivo-Conductual Grupal, que comprende las conductas violentas como aprendidas y representativas de mecanismos disfuncionales. Y en tercer lugar, la redefinición del rol masculino en el marco de las relaciones de pareja.

\section{Metodología}

\section{Tipo de Estudio}

El presente estudio, de tipo exploratorio-descriptivo, se basa en un enfoque cualitativo hermenéutico-interpretativo, ya que intenta identificar y analizar críticamente las concepciones de masculinidad desplegadas en el documento propuesto por el Ministerio del Interior para el trabajo con hombres que ejercen violencia.

\section{Muestra}

Para el establecimiento de la muestra documental se revisaron documentos gubernamentales emitidos por el Ministerio del Interior para el trabajo con hombres que ejercen violencia. Estos documentos fueron: "Intervención con hombres agresores en violencia contra la mujer" (Ministerio del Interior, 2008) e "Intervención con hombres que cometen actos de violencia en el contexto de las relaciones de pareja" (Ministerio del Interior, 2010). Siguiendo los objetivos del estudio se seleccionó la norma del año 2010, dado que contemplaba las directrices ya desarrolladas en el 2008. De esta forma, la muestra documental quedó finalmente constituida por el texto: "Intervención con hombres que cometen actos de violencia en el contexto de las relaciones de pareja" (Ministerio del Interior, 2010), contenidas en el Eje "Rehabilitación y reinserción social", correspondiente a las "Orientaciones administrativas y técnicas del fondo de apoyo a la gestión municipal”, planteadas por el Gobierno de Chile para el año 2010. 


\section{Técnica de análisis}

Se optó por realizar un análisis de contenido cualitativo entendido como una aproximación empírica, de análisis metodológicamente controlado de textos, que sigue reglas analíticas de contenido y modelos paso a paso sin cuantificación de por medio (Mayring, 2000, en Cáceres, 2003). La técnica de análisis de contenido seleccionada busca la obtención de resultados profundos e interpretativos más allá de los aspectos léxicogramaticales, o exclusivamente manifiestos (Pérez, 1994). El objetivo de la técnica, por tanto, fue develar las concepciones de masculinidad que otorgan sentido a la muestra documental.

\section{Procedimiento}

La primera etapa de la técnica de análisis de contenido propuesta por Cáceres (2003) postula la necesidad de explicitar la perspectiva y postulados teóricos que guiarán y en alguna medida influenciarán el análisis del material. De esta forma, se estableció como postura teórica, las contribuciones de los Estudios de la(s) Masculinidad(es) y los aportes de la Teoría Queer, con el objetivo de explicitar los supuestos a la base del trabajo. En este sentido, se rescataron los desarrollos teóricos en torno a la heteronormatividad que regula la constitución y relación entre los géneros, destacando así los significados asociados al hombre y a la masculinidad reproducidos por las distintas esferas de la vida social.

La segunda etapa consistió en la selección de extractos del texto o "unidades de análisis" (Cáceres, 2003) que tuvieran coherencia con los objetivos del estudio y el marco teórico. Debido a que el interés del estudio radica en el análisis de las concepciones de masculinidad a la base de las orientaciones técnicas de una intervención con hombres que ejercen violencia, se seleccionaron como unidades de análisis extractos o frases del texto que contuvieran las siguientes palabras: "hombres", "masculinidad/ es", "violencia” y "agresores". 
Por último, las unidades de análisis permitieron la construcción de categorías una vez que fueron sometidas a reflexión desde las coordenadas teóricas anteriormente expuestas.

\section{Resultados}

El análisis da cuenta de concepciones amparadas en la regulación heteronormativa de los sexos, donde prevalecen definiciones esencialistas, normativas y positivistas de la masculinidad. El texto revela argumentos que apelan a una crisis de la masculinidad, entendida como los cambios que a nivel del rol y la identidad han experimentado los hombres respecto al progresivo empoderamiento de la mujer.

Asimismo, las concepciones heteronormativas vertidas en las orientaciones técnicas dan cuenta de un tipo de masculinidad, y no de las diferentes articulaciones que dicha posición subjetiva puede asumir en base a diferentes variables. De esta forma, en las concepciones de masculinidad a las que hace alusión el documento es posible encontrar tres categorías:

\section{Concepciones esencialistas}

Los principios explicitados en las orientaciones técnicas reflejan concepciones esencialistas de la masculinidad, al destacar la ligazón existente entre la redefinición del rol masculino, los cambios propios de la modernidad, y la violencia de género. La rearticulación de la masculinidad -en singular- supondría un reordenamiento de rasgos inherentes a una masculinidad y, por tanto, propios de una forma particular de ser hombres. En otras palabras, la intervención circunscribe a la masculinidad histórica y culturalmente con cualidades asignadas a los hombres tal como se expresa en la siguiente cita: “...eventuales dificultades de los hombres para redefinir el rol masculino en un contexto social y cultural cambiante, en particular ante la constatación del progresivo empoderamiento que ha experimentado la mujer" (Ministerio del Interior, 2010, p. 223). 
Para el presente caso, los estereotipos de género se consolidan al contemplar el comportamiento agresivo como intrínseco a los hombres. Por ejemplo las orientaciones técnicas informan sobre una "Tipología de Agresores" (p. 243), en la que se incluyen tres subtipos: bajo, moderado, $o$ alto riesgo. También destacan: "Estos agresores inflingen el menor nivel de daño en sus parejas-víctimas" (Ministerio del Interior, 2010, p. 243).

\section{Concepciones normativas}

Por otra parte, dentro de las orientaciones técnicas también es posible identificar nociones normativas, al plantear la dificultad de algunos hombres para redefinir el "rol masculino" ante los cambios culturales acaecidos principalmente durante el siglo XX.

Al teñir su conceptualización de definiciones normativas, la intervención no contempla las transformaciones de la(s) masculinidad(es) en tanto posiciones subjetivas determinadas por condiciones de clase, históricas, étnicas, geográficas, por una parte; e inscribe el análisis de la masculinidad a "un" rol que debe ser rearticulado. Esto como se evidencia en el siguiente extracto:

Este proceso ha provocado que, de hecho, muchas mujeres logren ejercer de manera efectiva sus derechos, más allá de las expectativas de hombres socializados en el marco de una matriz cultural patriarcal, lo cual tensiona la forma en que estos hombres comprenden su propio rol en la relación de pareja (Ministerio del Interior, 2010, p. 223).

\section{Concepciones positivistas}

La noción positivista de masculinidad es reconocible dentro de las técnicas propuestas por la intervención -modelo cognitivo-conductual grupal- donde se propone la "corrección (...) de aspectos funcionales (pragmáticos) de la violencia", entendiendo que "las conductas violentas son aprendidas y representan mecanismos disfuncionales" (Ministerio del Interior, 2010, p. 223). Dicha corrección del comportamiento 
violento plantea una noción polarizada, quedando sujeta la masculinidad a tipificaciones de género donde habrían comportamientos funcionales y disfuncionales para los sujetos hombres que cometen actos de violencia (Connell, 1997). Así se revela en el siguiente párrafo: el “(...) 'tratamiento’ alude a un trabajo con alcances terapéuticos limitados al análisis y modificación funcional, orientado de manera pragmática a la corrección del comportamiento violento" (Ministerio del Interior, 2010, p. 221).

\section{Discusión y Conclusión}

La intervención en hombres que ejercen violencia contra la mujer en contextos de pareja propuesta por el Plan de Seguridad Pública del Ministerio del Interior para el año 2010, da cuenta de la tendencia evidenciada durante las últimas décadas hacia la proliferación de estudios e intervenciones que buscan abordar problemáticas vinculadas a las transformaciones del mundo contemporáneo y sus consecuencias en las relaciones genéricas: la permeabilidad de los límites entre lo público y lo privado; la crisis de la familia nuclear; la interpelación de valores patriarcales; las transformaciones en las formas de producción y en el flujo de la información, entre otras.

El despliegue de este tipo de iniciativas revela un avance importante respecto a lo realizado en Chile, dando cuenta de cambios significativos al abordar la disminución de la violencia contra la mujer, problema que si bien constituye un eje prioritario en la agenda de género del Estado, continúa siendo una deuda histórica no saldada y con repercusiones contingentes.

Como es posible constatar en los resultados del análisis, si bien las concepciones sobre la(s) masculinidad(es) se diferencian en su lógica normativas, esencialistas, positivistas-, estas no son claramente distinguibles al momento de encontrarlas (Connell, 1997). La falta de límites a la que se hace referencia, reflejaría la heteronormatividad hegemónica que regula la constitución de los géneros, que atribuye conceptos esencialistas y normativos a cada uno de los sexos (Butler, 1990). En otros términos, 
los significados ligados a lo femenino como propio de la mujer y a lo masculino como inherente al hombre.

Es precisamente esta distinción binaria -masculino/femenino- la que enmarca a los géneros en una construcción que se articula a partir de la diferencia anatómica de los sexos, desplazando ejes de poder partícipes en la conformación de los géneros: etnia, clase, entre otros (Butler, 2001). Las orientaciones técnicas de la intervención en hombres no poseen un concepto de masculinidad que contemple el hecho descrito por Butler (2000) acerca de que cualquier categoría de identidad controla, describe, autoriza y, en menor medida, libera; incluso si se trata de la identidad de ser "hombre" o "mujer". Por lo tanto, no existiría un género "masculino" propio del varón, y uno "femenino" que pertenece a las mujeres; el género es consecuencia de un sistema coercitivo que se apropia de los valores culturales de los sexos (Fonseca \& Quinteros, 2009).

La masculinidad, entendida desde la intervención, se concebiría necesariamente a partir del ser "hombre" - desde una perspectiva esencialista y normativa-, y no como una posición subjetiva inscrita en un entramado social complejo. Esto se expresaría en la ausencia de referencias en torno al ejercicio de la violencia sobre otras masculinidades consideradas subordinadas. Es decir, la intervención supone al hombre como esencialmente violento contra la mujer, y no a la posición masculina hegemónica -amparada en valores patriarcales- como devaluadora de lo femenino.

Si bien la violencia ejercida sobre masculinidades subordinadas -como es el caso de la población homosexual- podría parecer de menor importancia para las orientaciones técnicas, resulta relevante para el análisis que demanda el estudio de la(s) masculinidad(es) considerar que, desde los aportes de la Teoría Queer, un "hombre" es un homosexual que se niega a sí mismo (Butler, 2000), en tanto la represión de la homosexualidad masculina tiene como objetivo la conformación de la hombría y la estabilidad del sistema de géneros (Fonseca \& Quintero, 2009). En otros términos, el objeto que se reprime, y que posteriormente se violenta, sostiene y da cabida a aquellos rasgos que son atribuidos a los hombres. Esta perspectiva 
se distancia radicalmente de lo esencialista y normativo que reservan las concepciones de la masculinidad a la base de la intervención.

En cuanto al lugar que ocupa la propuesta del Plan de Seguridad Pública con respecto a otras iniciativas, cabe destacar cómo la definición de masculinidad mantiene el trayecto de reflexión sugerido por las intervenciones con varones en Chile. Como plantean Aguayo y Sadler (2010) los escasos esfuerzos han estado dirigidos fundamentalmente a temáticas de violencia, conceptualizando a los hombres como violentos, alcohólicos y delincuentes, sin reconocerlos como sujetos con vulnerabilidades psicosociales o necesidades en salud mental.

De esta forma, los supuestos establecidos en torno a la masculinidad convierten a los hombres en un objeto coherente para la política de seguridad pública, que busca reducir la violencia que se comete en espacios públicos mediante la intervención en hombres que llevan a cabo actos violentos en contextos privados, reduciendo la intervención -en gran medida- a un dispositivo de control. Una concepción de masculinidad entendida desde marcos hegemónicos, inscrita dentro de una normativa esencialista ligada a la heterosexualidad, posiciona al hombre en el lugar de lo potencialmente violento.

En este sentido, la clínica en cuestión se sostendría en un punto en el que convergen discursos tanto sanitarios como jurídicos, donde la "cura" de los sujetos se materializaría en la eliminación de los actos violentos en el contexto de pareja, y no necesariamente en la reelaboración de significados asociados a la posición con respecto no solo a la mujer, sino que también a otros hombres. El éxito de la intervención pasaría, por tanto, por la capacidad del sujeto de responsabilizarse de los actos cometidos, es decir, por la referencia a una norma de conducta y no por la tramitación de aspectos subjetivos involucrados en la violencia.

El encuentro entre ambos discursos devela una tensión que se expresa en las prácticas clínicas estipuladas por las orientaciones técnicas, las que se circunscriben al Eje de Rehabilitación y Reinserción Social, instalando una normatividad que resguardaría la seguridad pública, y restringiría el accionar clínico y las diversas expresiones de la masculinidad. Desde este marco, las intervenciones realizadas "en hombres" y no "con 
hombres" o "para hombres", dan cuenta de la no incorporación de las distintas dimensiones que involucran la conformación de la identidad, por una parte, y del control sobre el objeto a intervenir, por otra.

Sin embargo, si bien la intervención está enmarcada en un contexto de seguridad, y no en un discurso estrictamente sanitario, esta brinda el espacio terapéutico para que los hombres viertan su malestar, y así se posicionen como sujetos con necesidades y vulnerabilidades.

Asimismo, la intervención ha posibilitado instalar un discurso clínico -terapéutico- en un espacio de control proporcionado por el Plan de Seguridad Pública, permitiendo posicionar un abordaje interdisciplinario que da lugar a la palabra a quienes han sido silenciados por las políticas públicas en género: los hombres. Lo anterior radicaría en que el lenguaje empleado a partir de los modelos de intervención -que derivan de posturas epistemológicas diversas- devela una mixtura discursiva que atraviesa la problemática: el discurso clínico-psicológico, por una parte, y el jurídicocontrol, por otra.

Dicha superposición de discursos se expresa en que, si bien se explicita que la violencia contra la mujer no se asocia a la presencia de trastornos psiquiátricos en el hombre, y que por tanto, el trabajo psicoterapéutico no es estrictamente clínico, se busca el cambio concreto de la conducta violenta a través de prácticas clínicas correctivas. A la vez, la intervención se inscribe desde un marco o eje de trabajo que pretende "rehabilitar y reinsertar socialmente", situando a la intervención como dispositivo de control y seguridad.

La mixtura discursiva a la que se hace alusión se manifiesta concretamente en el trabajo que desarrollan los equipos interventores, donde la posición que ocupa la clínica queda adscrita al espacio de control propuesto por el Plan de Seguridad Pública. Si bien se puede entender como una desventaja para la intervención, invita a la reflexión de los equipos sobre el lugar que ocupan y las prácticas que desarrollan.

El enmarcar la intervención dentro de la política de seguridad pública, concibiendo a los hombres como intrínsecamente violentos y como un peligro potencial a desplegarse en lo social, da cuenta de un discurso que pretende -como objetivo último- controlar las consecuencias 
de la violencia a tal nivel. Así, el discurso de control emerge a partir de la concepción de violencia en el contexto íntimo como proclive a reproducirse en el ámbito público.

Las orientaciones técnicas consideran a un hombre inherentemente agresor, lo que genera contradicciones en la práctica de los interventores: la escucha de la violencia como síntoma a eliminar versus el malestar referido por los varones. Este obstáculo se constituye como una desventaja en el trabajo al representar al hombre como un sujeto sin vulnerabilidades y necesidades en salud mental.

Lo anterior da cuenta de una perspectiva esencialista y normativa de la masculinidad, la que limita el análisis al focalizar la problemática en comportamientos agresivos, y deja de lado ejes de poder en el que se articulan las identidades, a saber: raza, clase, etnia, y contexto sociocultural, entre otras. Circunscribir en forma totalizante la temática de la violencia en contextos de pareja, como una problemática del "rol masculino", obstruye la posibilidad de subvertir los mandatos hegemónicos y heteronormativos.

Por lo tanto, se considera que la concepción de masculinidad a la base de las orientaciones técnicas representa un sesgo histórico y cultural, limitándolo a atributos del hombre a partir de una concepción binaria de los sexos y de preceptos normativos impuestos por la masculinidad hegemónica. Dicha concepción, da lugar a prácticas discursivas que reproducen los mandatos de la heteronormatividad, excluyendo la posibilidad de entender a la masculinidad -más bien las masculinidades- en tanto posición subjetiva.

El discurso enunciado por las orientaciones técnicas no considera que la masculinidad hegemónica, sostenida en el patriarcado, ejerce violencia también sobre las masculinidades subordinadas (hombres gays, hombres indígenas, niños y ancianos). La dominación masculina y sus expresiones de violencia se enmarcarían en las relaciones de poder instituidas por la cultura patriarcal que devalúa los significados ligados a lo femenino. Bajo esta óptica, el hombre en sí no sería la causa de la violencia hacia la mujer, sino las relaciones de poder que en este caso actualiza. 
Las implicancias de hacer este giro en la reflexión permitirían la posibilidad de concebir al hombre como un sujeto con necesidades y vulnerabilidades, y no bajo una perspectiva esencialista que lo etiquete bajo los preceptos normativos. Frente a esto, los aportes de la perspectiva semiótica, el enfoque relacional de género y los estudios de las masculinidades a esta temática pueden nutrir su comprensión. El conceptualizar al hombre como un sujeto con conflictos y necesidades -cuestiones que la sociedad patriarcal desmiente- posibilita la instauración de la masculinidad en tanto posición subjetiva y no ligada a categorías de la heteronormatividad.

El discurso impulsado asienta la reproducción de oposiciones asimétricas entre femenino y masculino, donde la noción de (una) masculinidad -referida a los hombres- reproduce la idea de sexo del lenguaje hegemónico. A partir de esto, surgen interrogantes como: ¿Es siempre la pareja heterosexual? ¿Qué ocurre con la violencia en el contexto de pareja entre hombres y mujeres homosexuales? ¿Qué ocurre cuando es la mujer quien ejerce violencia hacia el hombre? ¿Dónde pueden acudir si las intervenciones tienen como base preceptos esencialistas?

A pesar de no ser la intención de este artículo dar una respuesta a estos cuestionamientos, sí se vuelve necesaria una aproximación que complejice y problematice la temática, abordando los distintos elementos partícipes. En este sentido, comprender las masculinidades desde definiciones semióticas -como los avances de la Teoría Queer- permitiría trascender la heteronormatividad -la dicotomía: hombre/mujer y masculino/femenino- para convertirse en una reflexión de género realmente relacional y contingente.

\section{Referencias}

Abarca, H. (2000). Discontinuidades en el modelo hegemónico de masculinidad. En: M. Gogna (Comp). Feminidades y Masculinidades: estudios sobre salud reproductiva y sexualidad en Argentina, Chile y Colombia (pp. 193-244). Buenos Aires: Cedes.

Aguayo, F. \& Sadler, M. (2010). The Man and Gender Equality Policy Project. En: What Men have to do with it: Public Policies to Promote Gender Equality (pp. 26-30). Promundo and International Center for Research on Woman. 
Amuchástegui, A. (2008). La verdad... yo no creía que era violento. Cuerpo emocional y subjetivación en un programa reeducativo para hombres violentos. En R. Parrini (Coord). Los Contornos del alma, los límites del cuerpo: género, corporalidad y subjetivación (pp. 155181). México.

Araujo, K. \& Rogers, F. (2000). El Hombre: ¿Existe? En J. Olavarría \& R. Parrini, (Eds). Masculinidades, identidad, sexualidad y familia (pp. 59-66), Santiago: Flacso-Chile.

Bonino, L. (2009). Micromachismos: La violencia invisible en las parejas. Red Iberoamericana de Masculinidades. Recuperado el 12 de mayo de 2010 de: http://www.redmasculinidades.com/resource/images/ BookCatalog/Doc/00068.pdf

Bourdieu, P. (2000). La dominación masculina. Buenos Aires: Anagrama.

Burín, M. (2007). Precariedad laboral, masculinidad, paternidad. En M. Burín, M. Jiménez Guzmán \& I. Meler (Comp). Precariedad laboral y crisis de la masculinidad. Impacto sobre las relaciones de género (pp. 33-44). Buenos Aires: Universidad de Ciencias Empresariales y Sociales (UCES).

Butler, J. (1990). Actos performativos y constitución de género: Un ensayo sobre fenomenología y teoría feminista. Revista Debate Feminista, $18,296-314$.

Butler, J. (2000). Imitación e insubordinación de género. Revista Occidente, 235, 85-109.

Butler, J. (2001). El género en disputa. El feminismo y la subversión de la identidad. México: Paidós.

Butler, J. (2006). Deshacer el género. Barcelona: Paidós.

Castells, M. (2001). La era de la información. Vol. 2: El Poder de la Identidad. México: Siglo XXI.

Cáceres, P. (2003). Análisis cualitativo de contenido: Una alternativa metodológica alcanzable. Psicoperspectivas, 2, 53-83.

Connell, R. (1997). La organización social de la masculinidad. En T. Valdés \& J. Olavarría, (Eds). Masculinidadles. Poder y crisis (pp. 31-48). Chile: Isis-Flacso.

De Keijzer, B. (1997). El varón como factor de riesgo: Masculinidad, salud mental y salud reproductiva. En E. Tunón (Coord). Género y salud en el Sureste de México (pp. 197-219). Villahermosa: Ecosur y UJAD.

De Keijzer, B. (2001). Hasta donde el Cuerpo Aguante: Género, Cuerpo y Salud Masculina. En C. Cáceres, M. Cueto, M. Ramos \& S. Vallenas (Coords). La salud como derecho ciudadano: perspectivas y propuestas desde América Latina (pp. 137-152). Lima: Universidad Peruana Cayetano Heredia. 
Fernández-Llebrez, F. (2005). Masculinidades y violencia de género. ¿Por qué algunos hombres maltratan a sus parejas (mujeres)? Red Iberoamericana de Masculinidades. Recuperado el 20 de mayo de 2010 de: http://www.redmasculinidades.com/resource/images/BookCata$\log /$ Doc/00052.pdf

Ferrer, V. \& Bosch, E. (2005). Introduciendo la perspectiva de género en la investigación sobre violencia de género. Revista Anales de psicología, 21(1), 1-10.

Fonseca, C. \& Quintero, M. L. (2009). La teoría Queer: la de-construcción de las sexualidades periféricas. Revista Sociológica, 69, 43-60.

Garda, R. (1998). Modernidad y Violencia de los Hombres. Reflexiones desde la Masculinidad sobre el Espacio-Tiempo y el Poder. Revista Electrónica La Ventana, 8, 174-206.

Ministerio del Interior (2010). Plan Comunal de Seguridad Pública. Orientaciones Administrativas y Técnicas del Fondo de Apoyo a la Gestión Municipal 2010. Gobierno de Chile.

Montecino, S. (1996). De lachos a machos tristes: la ambivalencia de lo masculino en Chile. En S. Montecino \& M. Acuña (Comps). Diálogos sobre el Género Masculino en Chile (pp. 19-27). Santiago: PIEG, Universidad de Chile.

Montesinos, R. (2002). Las rutas de la masculinidad. Ensayos sobre el cambio cultural y el mundo moderno. Barcelona: Gedisa.

Olavarría, J. (2000). De la identidad a la política: masculinidades y políticas públicas. Auge y ocaso de la familia nuclear patriarcal en el siglo XXI. En J. Olavarría \& R. Parrini (Eds). Masculinidad/es. Identidad, sexualidad y familia. Primer Encuentro de Estudios de Masculinidad (pp. 11-28). Santiago: Flacso-Chile/Universidad Academia de Humanismo Cristiano/Red de Masculinidad.

Olavarría, J. (2003). Los estudios sobre masculinidades en América Latina. Un punto de vista. Anuario Social y Politico de América Latina y el Caribe. Flacso/Unesco/ Nueva Sociedad, 6, 91-98.

Olavarría, J. (2008). Globalización, Género y Masculinidades. Las corporaciones transnacionales y la producción de productores. Nueva Sociedad, 218, 72-87.

Pérez, G. (1994). Investigación cualitativa. Retos e interrogantes. Tomo II. Técnicas de análisis de datos. Madrid: La Muralla S. A.

Real Academia Española. (2001). Diccionario de la lengua española. Vigésima Segunda Edición. Madrid: Espasa-Calpe S. A.

Sadler, M. (2007). Los hombres también se emocionan: hacia la construcción de masculinidades presentes. Gaceta de Psiquiatría Universitaria, 3(4), 437-449. 
Scott, J. (1997). El género, una categoría útil para el análisis histórico. En M. Lamas (Ed). El género: la construcción cultural de la diferencia sexual (pp. 265-302). México: Porrúa-Pueg.

Zarza, M. \& Froján, M. (2005). Estudio de la violencia doméstica en una muestra de mujeres latinas residentes en Estados Unidos. Revista Anales de psicología, 1(21), 18-26.

Fecha de recepción: 25 de agosto de 2010.

Fecha de aceptación: 15 de mayo de 2012. 